\title{
COMPOSIÇÃO DE CAROTENOIDES EM PASSIFLORAS DO CERRADO ${ }^{1}$
}

\author{
DANIELE CRISTINA WONDRACEK ${ }^{2}$, FÁBIO GELAPE FALEIRO 3 , SUELI MATIKO SANO 3 , \\ ROBERTO FONTES VIEIRA ${ }^{4}$, TÂNIA DA SILVEIRA AGOSTINI-COSTA ${ }^{4}$
}

RESUMO - O objetivo foi avaliar, por HPLC, a composição qualitativa e quantitativa de carotenoides em maracujás do cerrado. Frutos procedentes de acessos nativos de quatro espécies (Passiflora cincinnata, $P$. nitida, P. setacea e P. edulis) foram analisados, utilizando, como referência, o maracujá-amarelo comercial (P. edulis). As polpas de maracujá apresentaram neoxantina, violaxantina, cis-violaxantina, anteraxantina, luteína, zeaxantina, $\beta$-criptoxantina, poli-cis-caroteno, prolicopeno, cis- $\zeta$-caroteno, trans- $\zeta$-caroteno, trans$\beta$-caroteno, 13-cis- $\beta$-caroteno e fitoflueno. Em geral, os teores de carotenoides entre as espécies e entre os acessos da mesma espécie foram significativamente diferentes. A espécie $P$. edulis apresentou o maior número de carotenoides, com diferença entre os acessos. Em um acesso de P. edulis comercial, foi encontrado o trans- $\beta$-caroteno como o carotenoide principal $\left(7,8 \pm 0,8 \mu \mathrm{g} \mathrm{g}^{-1}\right)$ e no outro o trans- $\zeta$-caroteno $(11,4 \pm 0,4 \mu \mathrm{g}$ $\left.\mathrm{g}^{-1}\right)$. Dois acessos de $P$. edulis nativos do Cerrado apresentaram $c i s$ - $\zeta$-caroteno como carotenoide majoritário $\left(6,28 \pm 0,15 \mu \mathrm{g} \mathrm{g}^{-1}\right.$ e $12,1 \pm 0,7 \mu \mathrm{g} \mathrm{g}^{-1}$, casca amarela e roxa, respectivamente). O perfil de carotenoides em frutos de espécies de maracujá apresentou diversidade de composição, com potencial de uso para melhoramento genético para agregar maior valor ao produto e estimular o seu consumo.

Termos para indexação: Passiflora, composição bioquímica, compostos bioativos, recursos genéticos.

\section{CAROTENOID COMPOSITION IN CERRADO PASSIFLORAS}

\begin{abstract}
The objective was to evaluate by HPLC qualitative and quantitative carotenoids pulp composition of passion fruit. Fruits coming from native accessions of four Cerrado passion fruit species (Passiflora cincinnata, P. nitida, P. setacea and P. edulis) were analyzed using commercial passion fruit ( $P$. edulis) as reference. Passion fruit pulps showed neoxanthin, violaxanthin, cis-violaxanthin, antheraxanthin, lutein, zeaxanthin, $\beta$-cryptoxanthin, prolycopene, poli-cis-carotene, $c i s-\zeta$-carotene, trans- $\zeta$-carotene, trans$\beta$-carotene, 13-cis- $\beta$-carotene and phytofluene. In general, carotenoids content were significantly different among species and among accessions of the same species. P. edulis species showed greater number of carotenoids with differences among accessions. In one commercial passion fruit accession trans- $\beta$-carotene was the major carotenoid $\left(7.8 \pm 0.8 \mu \mathrm{g} \mathrm{g}^{-1}\right)$ and other was trans- $\zeta$-carotene $\left(11.4 \pm 0.4 \mu \mathrm{g} \mathrm{g}^{-1}\right)$. Two accessions of $P$. edulis native from the Cerrado showed cis- $\zeta$-carotene like the major carotenoid $\left(6.28 \pm 0.15 \mu \mathrm{g} \mathrm{g}^{-1}\right.$ and $12.1 \pm 0.7 \mu \mathrm{g} \mathrm{g}^{-1}$, yellow peel and purple peel, respectively). Characterization of carotenoids profile in passion fruit species fruits showed diversity of composition with potential use for genetic improvement to add greater value to product and encourage consumption.
\end{abstract}

Index terms: Passiflora, biochemistry composition, bioactive compounds, genetic resources.

\footnotetext{
'(Trabalho 075-11). Recebido em: 08-02-2011. Aceito em: 09-09-2011. Parte da dissertação apresentada pelo primeiro autor para obtenção de título de Mestre em Agronomia

${ }^{2}$ Bióloga, Embrapa Recursos Genéticos e Biotecnologia, Parque Estação Biológica, PqEB, W5 norte, final. CEP: 70770-900 Brasília-DF. Universidade de Brasília, Campus Universitário Darcy Ribeiro, CEP 70910-900, Brasília-DF. E-mail: daniele.wondracek@gmail.com ${ }^{3}$ Pesquisadores, Embrapa Cerrados, BR 020, Km 18, CEP: 73310-970 Planaltina-DF. E-mails: ffaleiro@cpac.embrapa.br; sueli@cpac.embrapa.br ${ }^{4}$ Pesquisadores, Embrapa Recursos Genéticos e Biotecnologia, Parque Estação Biológica, PqEB, W5 norte, final. CEP: 70770-900 Brasília-DF. E-mails: rfvieira@cenargen.embrapa.br; tania@cenargen.embrapa.br
} 


\section{INTRODUÇÃO}

O maracujá é muito consumido in natura e no preparo de doces, sucos e sorvetes, e como planta medicinal. Várias espécies nativas ocorrem no Cerrado e pelo menos cinco delas são chamadas de maracujá do Cerrado (BRAGA et al., 2010). O centro de distribuição geográfica é o Brasil (FALEIRO et al., 2006), mas falta conhecimento sobre as potencialidades da grande diversidade de espécies, que são muitas vezes subutilizadas.

Os carotenoides são pigmentos com coloração que varia entre o amarelo-claro e o vermelho e que se acumulam em cromoplastos de folhas, flores, frutos, raízes e sementes (VIEIRA; AGOSTINI-COSTA, 2007). Esses pigmentos atuam na fotossíntese, com a função de auxiliar na captação de energia luminosa e promover a proteção contra a foto-oxidação. No organismo humano, alguns carotenoides podem ser convertidos em vitamina $\mathrm{A}$, outros estão associadas à redução do desenvolvimento de doenças crônicodegenerativas devido ao potencial antioxidante que apresentam (BRITTON, 1995; AGOSTINI COSTA et al., 2010).

A composição de carotenoides em plantas varia tanto qualitativa quanto quantitativamente (AZEVEDO-MELEIRO; RODRIGUEZ-AMAYA, 2004). O perfil destes compostos foi avaliado em maracujá-amarelo e roxo comerciais (LEUENBERGER; THOMMEN, 1972; MERCADANTE et al., 1998; SILVA; MERCADANTE, 2002), contudo, ainda não foi determinado em espécies nativas de maracujá.

O objetivo deste estudo foi avaliar a composição qualitativa e quantitativa de carotenoides na polpa de espécies de maracujás do Cerrado com potencial agronômico, utilizando, como referência, o maracujá-amarelo comercial.

\section{MATERIAL E MÉTODOS}

Foram analisadas quatro espécies: Passiflora cincinnata Mast., conhecida como maracujá do cerrado, com polpa creme (acesso CPAC MJ-2601 redondo; acesso CPAC MJ-26-02 cabaça); $P$. nitida Kunth, conhecida como maracujá-suspiro, com polpa incolor (CPAC MJ-01-03); P. setacea D. C., conhecida como maracujá do sono, com polpa amarelo-clara (acesso CPAC MJ-12-01-BRS Pérola do Cerrado); acessos nativos de $P$. edulis Sims, polpa amarelo- escura (maracujá-amarelo CPAC MJ-36-01; maracujá-roxo CPAC MJ-21-01). Estes acessos utilizados foram coletados no Bioma Cerrado e, nos últimos 10 anos, têm sido conservados, cultivados e caracterizados em condições de campo, no Banco Ativo de Germoplasma da Embrapa Cerrados, Planaltina-DF. A área experimental possui o solo classificado como Latossolo Vermelho com textura argilosa (corrigido para acidez e nutrientes do solo) e está inserida no domínio morfoclimático do Cerrado, com clima tropical estacional (Aw), com uma precipitação média anual entre $1.400 \mathrm{~mm}$ e $1.600 \mathrm{~mm}$ e temperatura média anual entre $22^{\circ} \mathrm{C}$ e $27^{\circ} \mathrm{C}$. Um sistema de irrigação por gotejamento é utilizado na área experimental, principalmente ao longo período seco do ano na região do Cerrado. Os frutos comerciais de P. edulis Sims., empregados como referência, foram obtidos em hipermercado de Brasília-DF, em 2007 e 2008.

A extração de carotenoides (RODRIGUEZAMAYA, 2001) foi desenvolvida em ambiente com temperatura controlada, luminosidade reduzida, adição de antioxidante e incorporação de nitrogênio, para minimizar a degradação. Os carotenoides foram extraídos (cinco a seis extrações sucessivas) com acetona gelada e adição de hyflosupercel, como auxiliar de extração e filtração, e butil-hidroxitolueno (BHT), como antioxidante. Os carotenoides foram transferidos para o éter etílico, usando um funil de separação. Metade das amostras foi saponificada com KOH $10 \%$ em metanol, por uma noite (16 horas), à temperatura ambiente, e a outra metade das amostras não foi submetida a este processo. No dia seguinte, foi feita a lavagem do extrato com água destilada até $\mathrm{pH}$ neutro. $\mathrm{O}$ éter etílico foi evaporado a vácuo $\left(35^{\circ} \mathrm{C}\right)$ até quase secura. $\mathrm{O}$ resíduo foi seco em nitrogênio, diluído com hexano $(4 \mathrm{~mL})$, adicionado de BHT, acondicionado em vial,e o ar foi retirado com uma seringa de agulha fina. A tampa e o selo do vial foram envolvidos com parafilme, e o extrato foi mantido sob refrigeração $\left(-20^{\circ} \mathrm{C}\right)$ até o momento da injeção, feito dentro de sete dias. No momento da injeção, as amostras foram secas em nitrogênio, diluídas em acetona grau HPLC e filtradas em Millex LCR com membrana PTFE modificada 0,45 $\mu \mathrm{m} 13$ $\mathrm{mm}$ (Millipore).

A separação dos carotenoides foi realizada por cromatografia líquida de alta eficiência (HPLC), segundo Kimura e Rodriguez-Amaya (2002) e Azevedo-Meleiro e Rodriguez-Amaya (2004), utilizando-se de um cromatógrafo líquido de alta eficiência (Varian), equipado com detector de conjunto de diodos (PDA PS-335), bomba (PS-240), injetor automático (PS-410) e software Galaxie 1.9, em ambiente com temperatura controlada (22 \pm 01 ${ }^{\circ} \mathrm{C}$ ). Os carotenoides foram separados em coluna $\mathrm{C}_{18}$ ODS-2 $150 \times 4,6 \mathrm{~mm}, 3 \mu \mathrm{m}$ (Waters). A fase móvel utilizada foi acetonitrila (contendo $0,05 \%$ de 
trietilamina): metanol: acetato de etila em gradiente de 95:5:0, durante os 20 minutos iniciais da corrida, e 60:20:20 entre 20 e 60 minutos de corrida; o tempo de equilíbrio da coluna foi de 20 minutos. O fluxo utilizado foi $0,5 \mathrm{~mL} \mathrm{~min}^{-1}$, e o volume de amostra injetado foi $10 \mu \mathrm{L}$.

A identificação dos carotenoides foi realizada através dos espectros de absorção no UV-visível, fornecidos pelo detector de conjunto de diodos, pelo tempo de retenção, valores de $\mathrm{Rf}$ em camada delgada (utilizando éter de petróleo:acetato de etila na proporção 9:3 como fase móvel), cocromatografia com padrões e por reações químicas específicas. Os espectros foram adquiridos entre 500 e $360 \mathrm{~nm}$, e os cromatogramas foram processados no comprimento de onda máximo de absorção $\left(\lambda_{\max }\right)$ de cada carotenoide.

Os carotenoides foram quantificados através de curva de calibração externa e fator de correção, conforme Kimura e Rodriguez-Amaya (2002). O padrão de trans- $\beta$-caroteno foi fornecido pela DSM Nutritional Products (Suíça). Os demais padrões foram purificados conforme Rodriguez-Amaya (2001) por cromatografia em coluna aberta de óxido de magnésio (VETEC): hyflosupercel (Diacel) (1:2), a partir da polpa de maracujá-amarelo comercial. Os padrões purificados foram quantificados em espectrofotômetro Perkin-Elmer modelo lambda 25 a partir das respectivas absorbâncias máximas, utilizando-se dos valores de absortividade (RODRIGUEZ-AMAYA, 2001) e considerando-se a pureza de cada padrão avaliada por HPLC. O procedimento utilizado para a estocagem dos padrões purificados foi o mesmo realizado para o armazenamento das amostras.

$\mathrm{O}$ valor de vitamina A foi calculado a partir dos novos fatores de conversão de carotenoides próvitamínicos A, conforme U.S. Institute of Medicine (IOM, 2001). Foi realizada a comparação da concentração dos carotenoides e dos valores de vitamina A entre os acessos (3-4 repetições). Para a análise dos dados, utilizou-se o teste não paramétrico de KruskalWallis a um nível de significância de 5\%, com auxílio do software SAS versão 9.0 (2002).

\section{RESULTADOS E DISCUSSÃO}

Foram isolados nas polpas de maracujá 14 carotenoides: neoxantina, trans-violaxantina, cisviolaxantina, anteraxantina, luteína, zeaxantina, $\beta$-criptoxantina, prolicopeno, cis- $\zeta$-caroteno, trans$\zeta$-caroteno, trans- $\beta$-caroteno, 13 -cis- $\beta$-caroteno, fitoflueno e um poli-cis-caroteno não identificado (Tabela 1). O $\beta$-caroteno foi o carotenoide predo- minante na polpa de alguns acessos de maracujá;

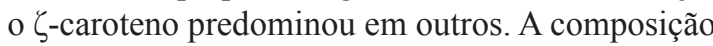
diferenciada de carotenoides nas polpas de maracujá está relatada em outros estudos. Em P. edulis roxo, Pruth e Lal (1958) encontraram fitoflueno, $\zeta$-caroteno, $\alpha$-caroteno e três carotenoides não identificados (MERCADANTE et al, 1998), mas Leuenberger e Thommen (1972) identificaram fitoflueno, $\zeta$-caroteno, $\beta$-caroteno, criptoxantina, auroxantina, mutatoxantina, $\beta$-apo- $12^{\prime}$-carotenal e $\beta$-apo-8' -carotenal, não mencionando $\alpha$-caroteno. Em P. edulis comercial, Mercadante et al. (1998) encontraram 13 carotenoides: fitoeno, fitoflueno, $\zeta$-caroteno (principal carotenoide), neurosporeno, $\beta$-caroteno, licopeno, prolicopeno, monoepóxi- $\beta$ caroteno, $\beta$-criptoxantina, $\beta$-citraurina, anteraxantina, violaxantina e neoxantina. Em maracujá-amarelo comercial, Silva e Mercadante (2002) encontraram também oito carotenoides: $\beta$-criptoxantina, prolicopeno, cis- $\zeta$-caroteno, $\zeta$-caroteno, $\beta$-caroteno e 13 -cis$\beta$-caroteno, além de neurosporeno e $\gamma$-caroteno em alguns lotes de frutos.

A espécie que apresentou o maior número de carotenoides foi P. edulis, destacando-se os dois acessos comerciais com 13 carotenoides (Figuras 1A-B), seguidos pelos acessos amarelo nativo (8 a 10 carotenoides, Tabela 1) e roxo nativo ( 9 carotenoides, Figura 1C). Na espécie P. setacea, foram encontrados sete carotenoides (Figura 1D). Os dois acessos de $P$. cincinnata apresentaram teor bastante reduzido de carotenoides. Em $P$. cincinnata 'cabaça', foram encontrados neoxantina, trans-violaxantina, anteraxantina, luteína, zeaxantina e trans- $\beta$-caroteno. Já no acesso 'redondo', foram encontrados os mesmos carotenoides, com exceção da neoxantina. A espécie $P$. nitida apresentou apenas traços de trans$\beta$-caroteno e de anteraxantina (Tabela 1).

O trans- $\beta$-caroteno e a anteraxantina, pertencentes à mesma rota biossintética, foram detectados em todos os acessos. A anteraxantina é um epóxido di - hidroxilado, derivado do $\beta$-caroteno. O 13-cis$\beta$-caroteno foi encontrado nos dois acessos de $P$. edulis comercial, em $P$. edulis amarelo e roxo nativos e em $P$. setacea. O cis- $\zeta$-caroteno foi detectado em todos os acessos de $P$. edulis e em $P$. setacea. $\mathrm{O}$ trans- $\zeta$-caroteno foi encontrado em todos os acessos de P. edulis. O poli-cis-caroteno, o prolicopeno, a $\beta$-criptoxantina e o fitoflueno foram encontrados nos acessos de $P$. edulis, tanto nativos quanto comerciais.

A neoxantina, que é um epóxido tri- hidroxilado derivado do $\beta$-caroteno, foi encontrada nos acessos de $P$. edulis comercial e no acesso $P$. cincinnata 'cabaça'. A cis- e a trans-violaxantina e a zeaxantina foram encontradas nos dois acessos de $P$. 
edulis comercial, em $P$. edulis amarelo nativo e em P. setacea. A trans-violaxantina também foi encontrada nos dois acessos de $P$. cincinnata (Tabela 1).

A luteína, identificada pela primeira vez no gênero Passiflora, foi encontrada apenas nas espécies nativas $P$. setacea e nos dois acessos de $P$. cincinnata. Esse dado é interessante, pois os acessos de $P$. edulis não possuem esse carotenoide di-hidroxilado, que apresenta um anel $\varepsilon$ e é derivado da rota biossintética dos $\alpha$-carotenoides, diferente dos demais carotenoides hidroxilados encontrados neste estudo, que são derivados do $\beta$-caroteno.

Foram quantificados neste estudo nove carotenoides: trans-violaxantina, cis-violaxantina, $\beta$-criptoxantina, prolicopeno, poli-cis-caroteno, cis- $\zeta$-caroteno, trans- $\zeta$-caroteno, trans- $\beta$-caroteno e 13-cis- $\beta$-caroteno (Tabela 1 ). Não foi realizada a comparação das concentrações dos carotenoides e dos valores de vitamina A em P. cincinnata 'redondo' e 'cabaça', P. nitida e $P$. setacea, devido às suas baixas concentrações, abaixo do limite de quantificação.

Os acessos comerciais de P. edulis apresentaram teores significativamente mais elevados $(\mathrm{p} \leq 0,05)$ de $\beta$-caroteno, de $\beta$-criptoxantina e de cis-violaxantina, em relação aos acessos nativos da mesma espécie, indicando um provável aumento de atividade das enzimas $\beta$-caroteno ciclase e $\beta$ caroteno hidroxilase em função do melhoramento genético, conforme sugerido por Silva e Mercadante (2002).

Entre os dois acessos de P. edulis comercial, foi encontrada uma inversão nos teores dos carotenoides majoritários; o trans- $\zeta$-caroteno foi encontrado em maior quantidade em $P$. edulis comercial a $\left(11,4 \pm 0,4 \mu \mathrm{g} \mathrm{g}^{-1}\right)$ e o trans- $\beta$-caroteno foi o carotenoide mais abundante em $P$. edulis comercial b $\left(7,8 \pm 0,8 \mu \mathrm{g} \mathrm{g}^{-1}\right)$. Nos dois acessos nativos de $P$. edulis, o cis- $\zeta$-caroteno foi o carotenoide majoritário $\left(6,28 \pm 0,15 \mu \mathrm{g} \mathrm{g}^{-1}\right.$ e $12,1 \pm 0,7 \mu \mathrm{g} \mathrm{g}^{-1}$, P. edulis amarelo e $P$. edulis roxo, respectivamente) (Tabela 1 ). Considerando a rota de carotenoides, esta condição pode ser explicada pela biossíntese de $\beta$-caroteno a partir do $\zeta$-caroteno, com introdução de novas duplas ligações e ciclização dos anéis, já que o acesso com maior teor de $\beta$-caroteno apresentou menor teor de $\zeta$-caroteno. Essa variação na proporção de carotenoides de maracujá também foi observada em outro trabalho (SILVA; MERCADANTE, 2002). Gama e Sylos (2005), Englberger et al. (2009), Kwasniewski et al. (2010) e Tadmor et al. (2010) também encontraram diferenças quantitativas de carotenoides em frutos. Variações quantitativas de carotenoides podem ser induzidas por fatores gené- ticos e ambientais, como luminosidade, temperatura, estação do ano, utilização ou não de agroquímicos (RODRIGUEZ-AMAYA, 2001).

Os valores de vitamina A calculados a partir do trans- $\beta$-caroteno variaram entre $0,04 \mu \mathrm{g} 100 \mathrm{~g}^{-1}$ de equivalente de atividade de retinol (RAE) em $P$. nitida e $65,4 \pm 4,9 \mu \mathrm{g} 100 \mathrm{~g}^{-1}$ RAE em um dos acessos de $P$. edulis comercial, refletindo a variação existente nas diferentes espécies de maracujá. Entre os acessos de $P$. edulis nativos e comerciais, essa variação foi de $21,4 \pm 0,9$ a $65,4 \pm 4,9 \mu \mathrm{g} 100 \mathrm{~g}^{-1}$ RAE. Nos acessos comerciais, possivelmente, devido ao processo de seleção para aumentar o teor de sólidos solúveis totais e a coloração da polpa, observou-se maior quantidade de carotenoides. Os valores de vitamina A apresentados nesta publicação estão de acordo com os novos fatores de conversão de vitamina A publicados pelo U. S. Institute of Medicine (IOM, 2001). Os valores para a conversão de carotenoides pró-vitamina A em vitamina A são: $1 \mu \mathrm{g}$ de equivalente de atividade de retinol $(\mathrm{RAE})=1 \mu \mathrm{g}$ de trans-retinol, $12 \mu \mathrm{g}$ de $\beta$-caroteno e $24 \mu \mathrm{g}$ de $\alpha$-caroteno ou $\beta$-criptoxantina (IOM, 2001). No trabalho de Silva e Mercadante (2002), os valores de vitamina A variaram de 43,63 $\pm 0,53$ a 244,50 $\pm 5,77$ ER $100 \mathrm{~g}^{-1}$. Porém, a fórmula utilizada no estudo considera $1 \mu \mathrm{g}$ de equivalente de retinol $(E R)=6 \mu \mathrm{g}$ de $\beta$-caroteno e bioatividade do trans- $\beta$-caroteno de $100 \%$, sendo esse valor o dobro do considerado para o $\beta$-caroteno pelo U.S. Institute of Medicine.

A ingestão média de vitamina A varia de 744$811 \mu \mathrm{g}$ RAE dia ${ }^{-1}$ para o homem e 530-716 $\mu \mathrm{g}$ RAE $\mathrm{dia}^{-1}$ para a mulher. Utilizando $\mu \mathrm{g}$ RAE, aproximadamente $26 \%$ da atividade de vitamina A consumida pelo homem e $34 \%$ pela mulher são provenientes dos carotenoides pró-vitamina A. Frutos coloridos, maduros e cozidos e tubérculos amarelos são mais eficientemente convertidos a vitamina $\mathrm{A}$ do que quantidades iguais de vegetais com folhas verdeescuras (IOM, 2001). Isso qualifica mais o consumo de maracujá pelo fato de o fruto conter $\beta$-caroteno e também $\beta$-criptoxantina, que são carotenoides com atividade pró-vitamina A e ação antioxidante. Portanto, tanto P. edulis comercial quanto P. edulis nativos podem ser fontes importantes de obtenção de carotenoides pró-vitamina $\mathrm{A}$ necessários à dieta e a uma alimentação saudável.

A variação na composição qualitativa e quantitativa de carotenoides entre as espécies e entre os acessos de Passiflora indica que a caracterização do perfil deste pigmento na polpa de maracujá com potencial agronômico pode agregar valor ao produto silvestre e estimular o seu consumo. 


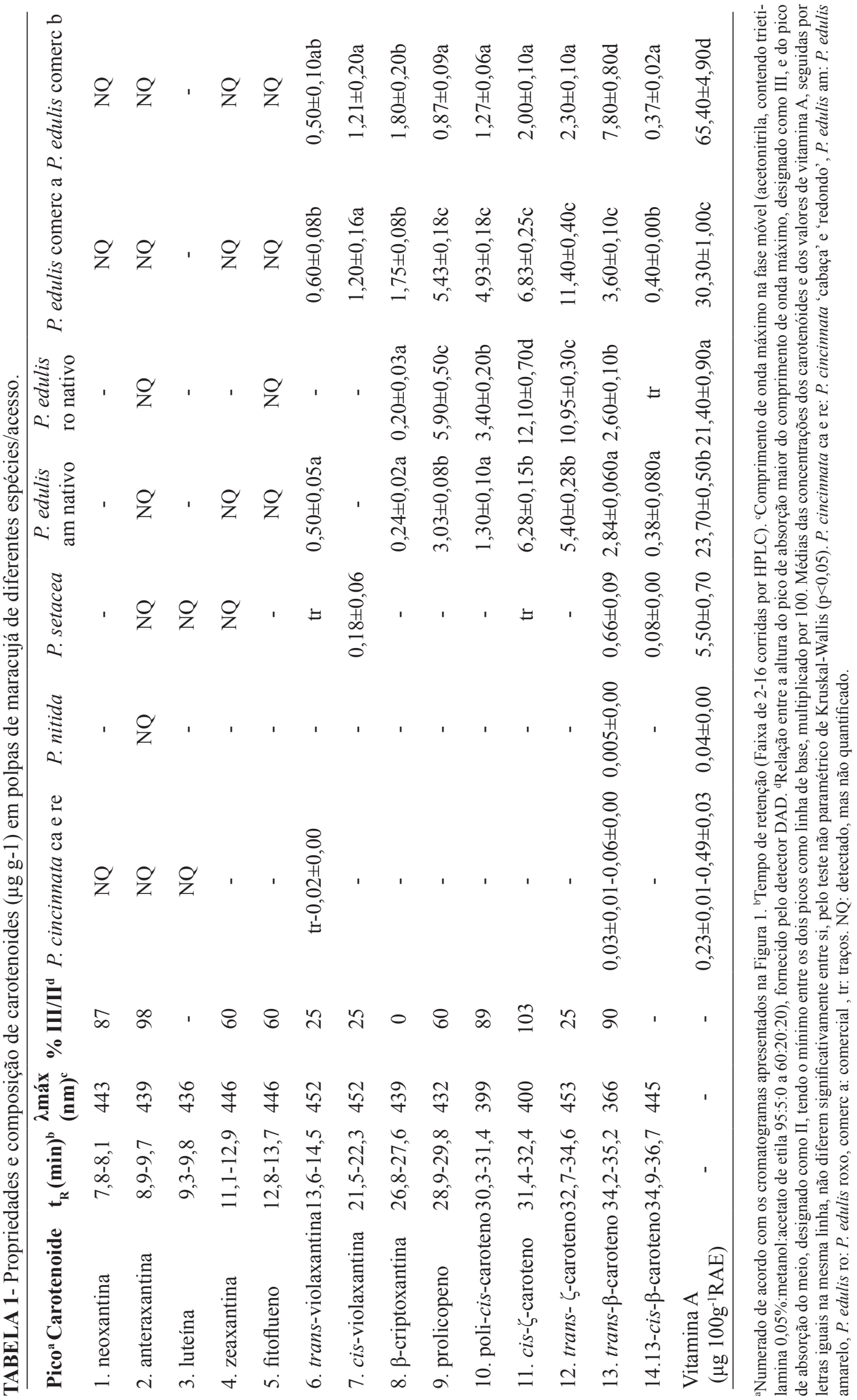



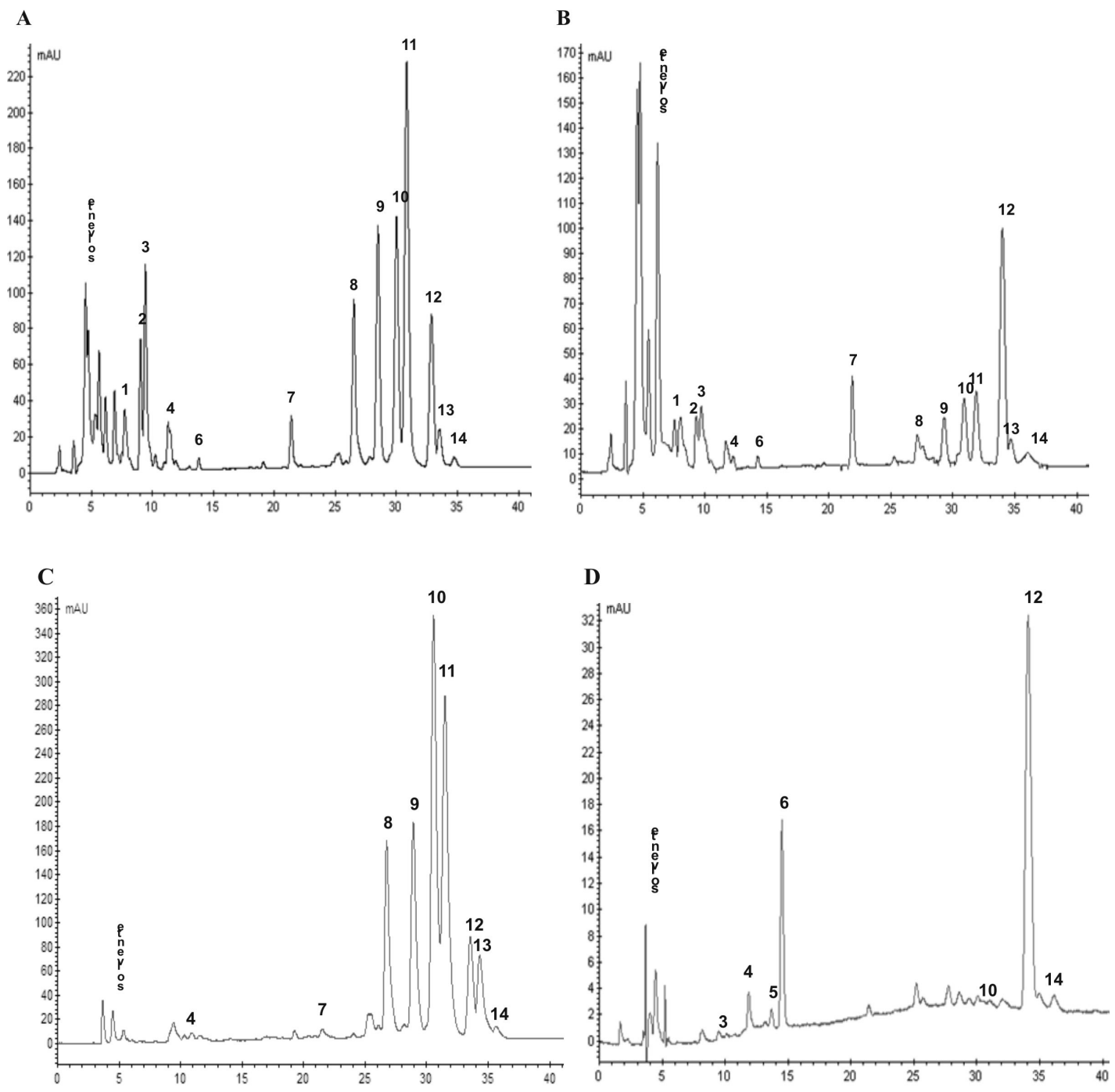

FIGURA 1 - Cromatogramas obtidos por HPLC: A) Passiflora edulis comercial a, com elevado teor de cise trans- $\zeta$-caroteno (picos 10 e 11). B) P. edulis comercial b, com elevado teor de $\beta$-caroteno (pico 12). C) P. edulis roxo nativo. D) P. setacea. Nas figuras $1 \mathrm{~A}, 1 \mathrm{~B}, 1 \mathrm{C}$ e $1 \mathrm{D}$, os números representam: 1. Neoxantina; 2. Trans-violaxantina; 3. Cis-violaxantina; 4. Anteraxantina; 5. Luteína; 6. Zeaxantina; 7. $\beta$-criptoxantina; 8. Prolicopeno; 9. Poli-cis-caroteno; 10. Cis- $\zeta-$ caroteno; 11. Trans- $\zeta$-caroteno; 12. Trans- $\beta$-caroteno; 13 . Fitoflueno; 14 . 13-cis- $\beta$-caroteno.

\section{CONCLUSÃO}

A variação na composição qualitativa e quantitativa de carotenoides entre as espécies e os acessos de Passiflora indica que a caracterização do perfil deste pigmento na polpa de maracujá com potencial agronômico pode agregar valor ao produto silvestre e estimular o seu consumo.

\section{AGRADECIMENTOS}

À Empresa Brasileira de Pesquisa Agropecuária, ao Conselho Nacional de Desenvolvimento Científico e Tecnológico, ao Programa Biodiversidade Brasil-Itália e ao Programa Agrofuturo, pelo suporte financeiro. 


\section{REFERÊNCIAS}

AGOSTINI COSTA, T. S.; WONDRACEK, D. C.; LOPES, R. M.; VIEIRA, R. F.; FERREIRA, F. R. Composição de carotenoides em canistel (Pouteria campechiana (Kunth) Baehni). Revista Brasileira de Fruticultura, Jaboticabal, v. 32, p. 903-906, 2010.

AZEVEDO-MELEIRO, C.H.; RODRIGUEZAMAYA, D.B. Confirmation of the identity of the carotenoids of tropical fruits by HPLC-DAD and HPLC-MS. Journal of Food Composition and Analysis, San Diego, v. 17, p. 385-396, 2004.

BRAGA, M. F.; JUNQUEIRA, N. T. V.; FALEIRO, F. G.; BERNACCI, L. C. Maracujá-do-Cerrado. In: VIEIRA, R. F.; AGOSTINI COSTA, T. S.; SILVA, D. B.; FERREIRA, F. R.; SANO, S. M. (Ed.). Frutas nativas da região centro-oeste do Brasil. Brasília: Embrapa Recursos Genéticos e Biotecnologia, 2010. p. 247-264.

BRITTON, G. Structure and properties of carotenoids in relation to function. The FASEB Journal, Bethesda, v. 9, p. 1551-1558, 1995.

ENGLBERGER, L.; SCHIERLE, L.; HOFMANN, P.; LORENS, A.; ALBERT, K.; LEVENDUSKY, A.; PAUL, Y.; LICKANETH, E.; ELYMORE, A.; MADDISON, M.; DEBRUM, I.; NEMRA, J.; ALFRED, J.; VELDE, N. V.; KRAEMER, K. Carotenoid and vitamin content of micronesian atoll foods: Pandanus (Pandanus tectorius) and garlic pear (Crataeva speciosa) fruit. Journal of Food Composition and Analysis, San Diego, v. 22, p. 1-8, 2009.

FALEIRO, F. G.; PEIXOTO, J. R..; PIO VIANA, A.; BRUCKNER, C.; LARANJEIRA, F. F.; DAMASCENO, F.; MELETTI, L. M. M.; CONSOLI, L.; SOUSA, M. A. F.; SILVA, M. S.; PEREIRA, M. G.; STENZEL, N.; SHARMA, R. D. Demandas para as pesquisas relacionadas ao melhoramento genético. In: FALEIRO, F. G.; JUNQUEIRA, N. T. V.; BRAGA, M. F. (Ed.). Maracujá: demandas para a pesquisa. Planaltina: Embrapa Cerrados, 2006. p. 25-34.

GAMA, J. J. T.; SYLOS, C. M. Major carotenoid composition of Brazilian Valencia orange juice: Identification and quantification by HPLC. Food Research International, Barking, v. 38, p. 899-903, 2005.

IOM - Institute of Medicine Food and Nutrition Board. Dietary reference intakes: for vitamin a, vitamin $\mathrm{k}$, arsenic, boron, cromium, copper, iodine, iron, manganese, molybdenium, nickel, silicon, vanadium and zinc. Washington: National Academy Press, 2001. 773 p.
KIMURA, M.; RODRIGUEZ-AMAYA, D.B. A scheme for obtaining standards and HPLC quantification of leafy vegetable carotenoids. Food Chemistry, London, v. 78, p. 389-398, 2002.

KWASNIEWSKI, M. T.; HEUVEL, J. E. V.; PAN, V. S.; SACKS, G. L. Timing of cluster light environment manipulation during grape development affects $\mathrm{C} 13$ norisoprenoid nd carotenoid concentrations in riesling. Journal of Agricultural and Food Chemistry, Easton, v. 58, p. 6841-6849, 2010.

LEUENBERGER, F. J.; THOMMEN, H. Zum Vorkommen von carotinoiden in der Passionsfrucht. Zeitschrift fur Lebensmittel-Untersuchung UndForschung, Berlim, v.19, p. 279-282, 1972.

MERCADANTE, A. Z.; BRITTON, G.; RODRIGUEZ-AMAYA, D. B. Carotenoids from Yellow Passion Fruit (Passiflora edulis). Journal of Agricultural and Food Chemistry, Washington, v. 46, p. 4102-4106, 1998.

RODRIGUEZ-AMAYA, D.B. A guide to carotenoid analysis in food. Washington: International Life Sciences Institute, 2001. 64 p.

SAS INSTITUTE. Statistical analysis system for Windows v.9.0. Cary, 2002.

SILVA, S. R; MERCADANTE, A. Z. Composição de carotenoides de maracujá-amarelo (Passiflora edulis flavicarpa) in natura. Ciência e Tecnologia de Alimentos, Campinas, v. 22, p. 254-258, 2002.

TADMOR, Y.; BURGER, J.; YAAKOV, I.; FEDER, A.; LIBHABER, S. E.; PORTNOY, V.; MEIR, A.; TZURI, G.; SA'AR, U.; ROGACHEV, I.; AHARONI, A.; ABELIOVICH, H., SCHAFFER, A. A.; LEWINSOHN, E.; KATZIR, N. Genetics of Flavonoid, Carotenoid, and Chlorophyll Pigments in Melon Fruit Rinds. Journal of Agricultural and Food Chemistry, Easton, v. 58, p. 10722-10728, 2010.

VIEIRA, R. F.; AGOSTINI-COSTA, T. S. Caracterização química de metabólitos secundários em germoplasma vegetal. In: NASS, L. L. (Ed.). Recursos genéticos vegetais. Brasília: Embrapa Recursos Genéticos e Biotecnologia, 2007. p. 343-372. 